

\title{
Design and development of ankle-foot prosthesis with delayed release of plantarflexion
}

\author{
Michael Mitchell, MSc; ${ }^{1}$ Katelynn Craig, MSc; ${ }^{1}$ Peter Kyberd, PhD; ${ }^{*}$ Edmund Biden, DPhil; ${ }^{2}$ Greg Bush, CP${ }^{1}$ \\ ${ }^{1}$ Institute of Biomedical Engineering, University of New Brunswick, Fredericton, New Brunswick, Canada; ${ }^{2}$ Mechanical \\ Engineering and School of Graduate Studies, University of New Brunswick, Fredericton, New Brunswick, Canada
}

\begin{abstract}
A computer-controlled mechanism that fits a standard ankle-foot prosthesis was designed to capture the absorbed energy in the ankle and delay its release until specific times in the gait cycle. This mechanism used a direct current motor to take up and hold the compression of a carbon-fiber ankle joint. Based on the timing of the contact forces between the foot and the ground, a microprocessor released the spring at preset times later in the gait cycle. This mechanism was added to a Talux prosthetic foot and was employed by a user of a conventional energy-storage ankle-foot prosthesis. His gait was recorded using a motion analysis system. Five settings: 0, 55, 65,75 , and 85 ms delay were tested on separate days, and the standard kinematic and kinetic gait data were recorded. The user reported some settings were more comfortable than others. When these preferences were tested with a randomized doubleblind trial, the preferences were not consistent. A second user showed a preference for the $55 \mathrm{~ms}$ delay. The modifications to the device resulted in changes to the gait of the subjects, including increased cadence and kinematics of the unaffected joints and a longer, slower push from the ankle, which was noticed by both of the subjects.
\end{abstract}

Key words: ankle, artificial legs, energy-storage prostheses, intelligent control, microprocessor control, motion analysis, prosthesis, prosthetic feet, prosthetic limbs, rehabilitation.

\section{INTRODUCTION}

The design and construction of limb prostheses have evolved from simple wooden pegs and metal hooks to computer-controlled devices. Studies have shown that the body adapts to the changes in gait induced by the loss of a limb [1-3]. Biological models have been developed that assist in the study of the forces and geometries that are present in gait and allow analysis of the motion. Thus, the changes in the kinematics and kinetics of human gait are broadly understood, both in the general population and for users of prosthetic limbs [4-5]. One critical aspect of human gait is the work performed by the triceps surae between heel-off and toe-off (TO). It is responsible for approximately 80 percent of the energy output of the walking cycle [6]. Persons with transtibial absences who use prosthetic ankles lack this capacity to actively generate energy. Current clinically employed prosthetic anklefeet are energy-storage-and-return designs that do not generate any additional power [7-11]. There has been recent interest in investigating designs that supply energy at the ankle, but the requirements for generating and storing the energy make this a difficult problem [12]. Development of powered prosthetic ankles remains a challenge; they need to be compact enough to be used by a wide range of patients with sufficient power to be useful, while light enough to be practical.

\footnotetext{
Abbreviations: DC $=$ direct current, FSR $=$ force-sensing resistor, $\mathrm{TO}=$ toe-off.

*Address all correspondence to Peter Kyberd, PhD; Institute of Biomedical Engineering, University of New Brunswick, 25 Dineen Dr, PO Box 4400, Fredericton, NB E3B 5A3; 506-453-4966; fax: 506-453-4827.

Email: pkyberd@unb.ca

http://dx.doi.org/10.1682/JRRD.2011.06.0107
} 
For a powered prosthetic device to be useful, it must generate sufficient power to have an effect on gait. The ankle can generate peak power of $3( \pm 1) \mathrm{W} / \mathrm{kg}$ during nondisabled gait [13]. For a powered ankle to be comparable, it would have to deliver the same energy quickly from an actuator and the user would have to carry batteries with sufficient energy storage to be useful over an extended period (e.g., an entire day). Previously, devices were completely incapable of generating sufficient power to be useful. In recent years, however, the technology has approached practical levels of performance and it has started to become conceivable that the goal of a suitable powered prosthetic ankle may be achieved. Klute et al. produced a powered ankle capable of adding energy to gait, but it was pneumatic [14]. Such systems are less feasible as energy storage for prosthetic limbs. For example, recharging gas storage is generally not practical [15]. More recent work has focused on electrically powered ankle units [16-17]. It is significant that both electrical and pneumatic systems use energy storage with series elastic elements. Springs form an important part of the method. They allow deployment of sufficient energy at the right moment in the gait cycle. Even then, the increase in mass, size, or cost that might be incurred may preclude their use by some persons with a loss or absence of a foot and ankle. Thus, investigating other aspects of prosthetic gait is still worthwhile.

Conventional devices use the elastic properties of materials, such as carbon fiber and rubber, to absorb the force of impact and to release it at the end of the stride, thus yielding a propulsive force. The timing of the energy release is entirely controlled by the unweighting of the foot, returning all of the energy at the time and at the rate of the TO of the prosthesis. These devices are highly successful in providing a more active lifestyle for users. However, it is possible that adjusting the timing of the energy release may provide a smoother prosthetic gait. Even if this is not the case, altering the release time could provide further insight into prosthetic gait by providing the propulsive force at specific times during the gait cycle. The purpose of this study was to investigate prosthetic gait and to determine whether release of the foot's absorbed energy at controlled times had an impact on the gait cycle. It was hypothesized that the delay would be felt by the subject. It was unknown whether this would be a welcome or unwelcome change. One foot design uses a coil spring and a microprocessor to capture the energy of the heel strike and return it for TO [18]. Early experiments show reduced energy expenditure by the subject and greater TO power from the prosthesis [19-20]. However, at the time of this writing, the systematic exploration of timing of the release with this device has yet to be reported in the literature.

\section{DESIGN}

An Össur (Reykjavik, Iceland) Talux foot (size $26 \mathrm{~cm}$, mass $500 \mathrm{~g}$, weight range 89 to $100 \mathrm{~kg}$ ) was chosen as the basis of the modified prosthesis. The Talux is aimed at an activity level of 5 (moderate). The prosthesis was modified to capture the maximum deflection induced on the foot during dorsiflexion and retain it for a controlled period of time. Figure 1 shows the gait cycle for an unmodified prosthesis. At heel strike, the ankle plantarflexes and the distance between the tibial component

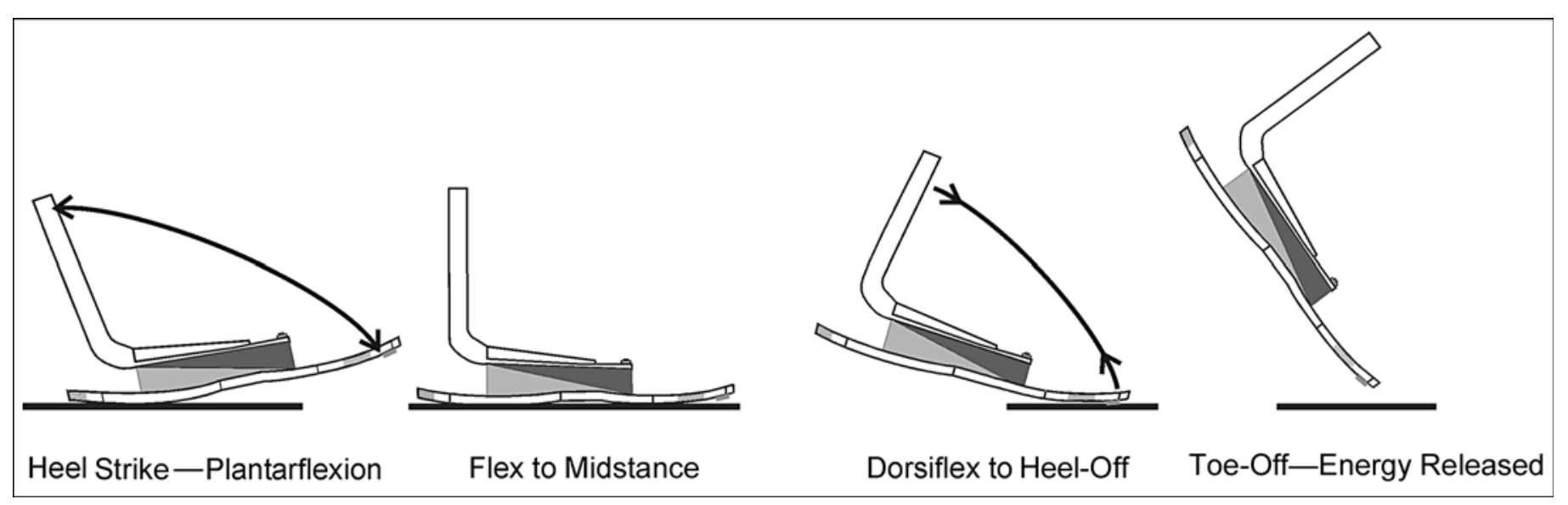

Figure 1.

Schematic of energy-storage mechanism of ankle-foot prosthesis. 
and the toes increases. As the user progresses to the stance phase, the ankle flexes. During dorsiflexion, the body's center of mass is directly above the foot's center of pressure and the foot is compressed. This decreases the distance between the shank and the toe. For the design described here, this displacement was captured by the mechanism and released later in the gait cycle (Figure 2). The shank and toe were linked using Spectralon fibers; by holding them at their shortest length, the system was able to retain the foot's absorbed energy. By winding the fibers around an axle, the system ensured the linear ground reaction forces were converted to a torque. A permanent magnet direct current (DC) motor then applied a counter torque to hold the fibers and so the ankle. The motor was used as an electromechanical brake-short circuiting the coils of the motor, which resisted the torque, stored energy. By removing the short circuit, the system controlled the release of the fibers, returning the foot to equilibrium after each successive step. A spring and one-way clutch were used to wind up the fibers. The addition of an over-running clutch allowed the spring to maintain tension on the line without needing to overcome the motor's rotational friction. This rotational friction does not absorb

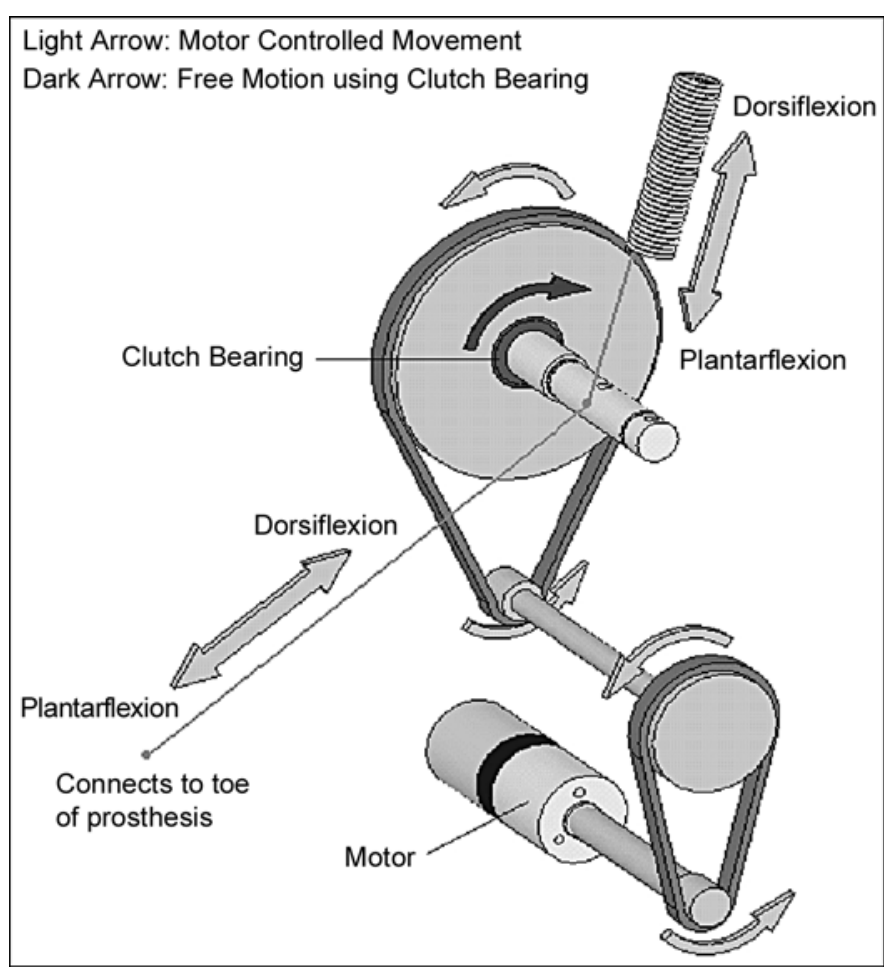

Figure 2.

Schematic of energy-storage mechanism of system. appreciable energy that would have been returned to the ground, it merely slows the release. Its effect on the release rate was the same for all delay settings.

The chosen actuator for the system was a 2224 series $(6 \mathrm{~V}, 4.5 \mathrm{~W})$ permanent magnet DC motor (Faulhaber Motors; Sachseln, Switzerland) with a 20/1 microplanetary gearbox and a 66:1 reduction ratio. With two subsequent levels of reduction, the torque acting on the motor was $0.54 \mathrm{Nm}$. The motor with gearbox had a recommended maximum torque output of $0.7 \mathrm{Nm}$, which was sufficient to hold the tension on the foot. A schematic of the electronics is shown in Figure 3. The controller, a PIC18F4680 (Microchip Technology Inc; Chandler, Arizona) with motor driver, was powered by an onboard battery and received data from a force-sensing resistor (FSR) (Interlink Electronics, Inc; Camarillo, California) positioned at the toe of the prosthesis. The microprocessor controlled the storage and release of the tension; it used a $20 \mathrm{MHz}$ clock and had an analog conversion rate of $2 \mathrm{kHz}$. The location of the FSR was determined empirically to ensure that the toe sensor could record the moment of maximum dorsiflexion. The FSR was mounted rigidly to the cosmetic shell of the foot, making a firm base. At the same time as the trials, the unmodified foot was fitted with the FSR. Through repeated tests, it was observed to trigger at the same point in the stride with no failures or false triggering. The FSR at the toe recorded the start of toe contact. When measured, the signal from the FSR rose rapidly to a plateau in about $50 \mathrm{~ms}$ before the recorded force dropped back to 0 after about $120 \mathrm{~ms}$. Thus, the range of roughly 50 to $120 \mathrm{~ms}$ represents when the output of the FSR was

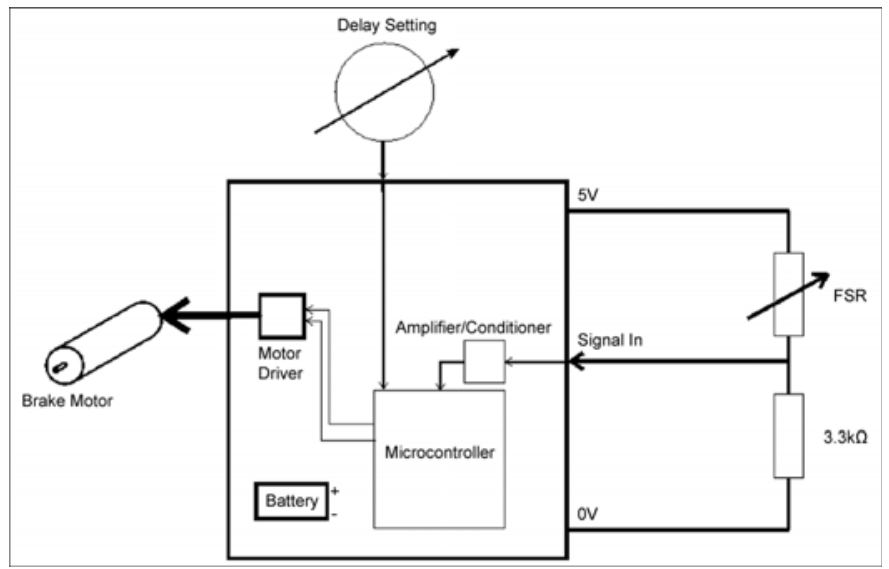

Figure 3.

Schematic of microprocessor/electronics. FSR = force-sensing resistor. 
uniform and so is a reliable signal from which to judge the timing. Therefore, $55 \mathrm{~ms}$ was chosen as the start; $120 \mathrm{~ms}$ was deemed to be too late. Hence, the delays were chosen to operate within this window. At full compression, the controller engaged the electromechanical brake, delaying the prosthesis' plantarflexion for a predetermined time before releasing the brake.

The total mass of the modified prosthesis, including battery and motor, was $950 \mathrm{~g}$. The mechanism replaced the existing prosthesis' male pyramid adapter and attached to the carbon fiber upright (Figure 4).

One consequence of the design was an increase in the mass of the entire prosthesis. There have been a number of studies investigating the impact of increased mass on the gait of transtibial prosthesis users. Selles et al. [21] and Royer and Martin [22] found little effect on kinematics, while Mattes et al. [23] found some changes to the kinematics. One conclusion made by Rusaw and Ramstrand [24] in their literature review was that most experiments used small sample sizes, which might explain the lack of consistent findings. Gailey et al. found an increase in the oxygen used and a slowing of the gait in a larger population (39 transtibial subjects) [25]; this represents one of the largest studies to date, but it did not record more detailed kinematic data (having been conducted earlier, in 1994). It is therefore hard to be certain what impact the increased mass of the braking mechanism would have on the gait; the variability of the data imply that any effects caused by the change in mass are small and may well be swamped by other differences.

\section{OPERATING CHARACTERISTICS}

The following is a description of the operational characteristics of the foot between the moment before heel strike and TO (Figure 1).

\section{Stage 1: Heel Strike to Foot Flat-Plantarflexion}

As the foot came into contact with the ground, the fibers connecting the toe of the prosthetic device to the shank were put in tension. The direction of rotation caused by the linear force acted against the over-running clutch and applied the load to the system. The electric

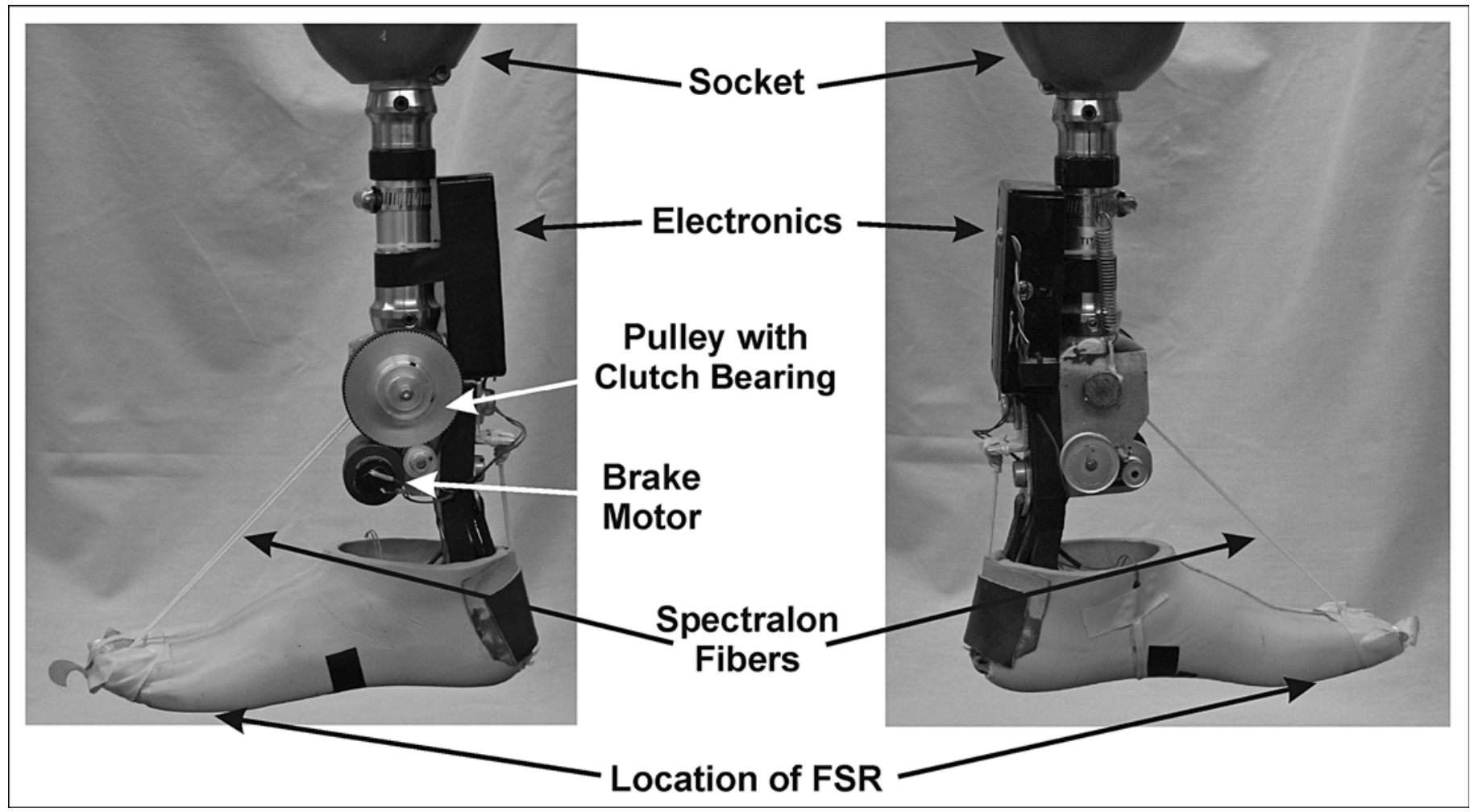

Figure 4.

Energy-storage mechanism on prosthesis. FSR = force-sensing resistor. 
motor was not powered and was allowed to spin freely, thus lowering the foot to the ground.

\section{Stage 2: Dorsiflexion to Midstance}

As the shank began to rotate over the ankle, the tension in the fibers was maintained via a spring. The shortening distance between the toe and the shank allowed the spring, acting via the over-running clutch, to take up the slack in the fibers. The electric motor was engaged once the toe FSR made contact with the ground.

\section{Stage 3: Dorisflexion Heel-Off and Toe-Off}

The fibers were put in tension as the carbon fiber foot attempted to return to its nondeformed position. The direction of rotation caused by the fibers' tension acted against the over-running clutch and applied the load to the system. Using the shorted electric motor as a brake, the microprocessor controlled the point of energy release. This release occurred at a fixed interval after the toe FSR recorded contact with the ground. For the tests, the delay values were selected to give a wide range of different responses.

\section{Stage 4: Plantarflexion: Toe-Off-Energy Released}

Immediately after TO, the foot dorsiflexed to return to steady state. Fiber tension was maintained by the shank-mounted spring acting with the over-running clutch until it was released by the microprocessor.

\section{CASE STUDY}

\section{Subjects}

The device was tried by two individuals. They were chosen based on having similar levels of experience. Use of the same device also limited the subjects' size and mass to the same characteristics. The first subject was involved in the initial design phase and so had full gait analysis; the second simply tested the device and the delay settings. The first subject had more than $20 \mathrm{yr}$ experience wearing an ankle-foot prosthesis. He was fitted with the foot using his conventional patellar-tendon-bearing transtibial socket with sleeve suspension over his residuum, which was $15 \mathrm{~cm}$ below the knee center. The foot was conventionally aligned to provide comfortable gait. The subject was $170 \mathrm{~cm}$ tall and weighed $89 \mathrm{~kg}$; he had lost his limb through trauma. Full motion analysis of his gait was performed.

A second subject was later fitted with the ankle-foot to his usual socket and was only tested while walking with the prosthetic system with different release delay times. The nature of the ankle-foot, i.e., a fixed size and spring rate, meant that the second subject had to be of similar height, mass, and amputation side; the second subject also lost his leg through trauma and had a similar level of experience with his device. Neither used any walking aids or had a history of falls. They were active users of their prostheses, as determined by self-report and the service history of their prostheses. Neither had any known neurological deficits.

\section{Protocol}

Subject 1's gait was recorded while he walked with the experimental prosthesis unmodified. Subsequently, the modified device was tested with five different delay settings ( $0,55,65,75$, and $85 \mathrm{~ms})$. A session consisted of fitting the subject with the prosthesis, then providing him time to adjust to the setting before recording 20 progressions through the motion capture system. One setting was investigated at each session; thus, he returned for five sessions. Motion analysis was performed using an eightcamera VICON M-Cam system (Vicon; Oxford, United Kingdom) and four Kistler force platforms (Kistler Instrument Corp; Amherst, New York). The two systems were synchronized, and the force plate data were collected at $600 \mathrm{~Hz}$, while the motion analysis data were collected at $60 \mathrm{~Hz}$.

Marker balls were placed on the sacrum, anterior superior iliac spine, greater trochanter, lateral femoral condyle, lateral malleolus, heel, and 5th metatarsal head on each leg (Figure 5). The subject was instructed to walk across the force plates at a self-selected speed. A successful trial was achieved when two complete consecutive stance phases were recorded on two plates. Subject 1 using the foot is shown in Figure 5.

Following the sixth measurement session, after all settings were tested, the subject returned for a final session during which all the different settings were presented in a random order. The values of the delays were not disclosed to the tester or the subject; thus, the test was randomized and double blind.

\section{Subject 2}

This trial consisted of a single session during which the subject was fitted with the limb and allowed to adjust to each setting before a video recording was made of him walking with the limb. The delays were presented in a random order, as for Subject 1, and both tester and subject were once again blinded to the length of the delay. 


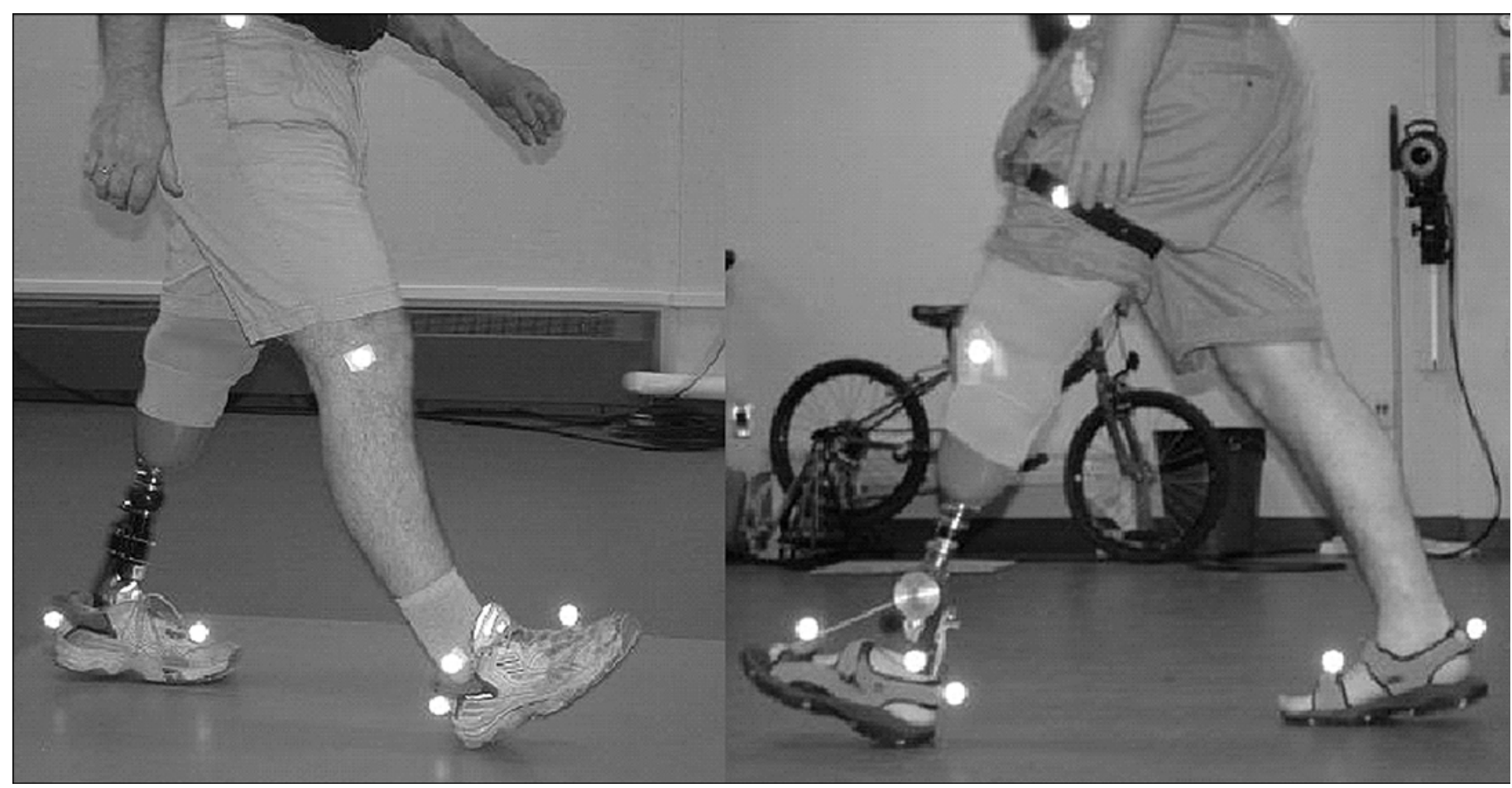

Figure 5.

Subject 1 wearing modified ankle-foot prosthesis, showing marker system used.

\section{Marker Placement}

Three markers were placed on the prosthesis. The toe and heel markers were placed on the shoe; because they were on the contralateral side and the conventional model assumes a semirigid natural foot, the impact on the results was likely minimal. The third marker was on the lateral malleolus of the intact foot; this is the standard approximation for the axis of the ankle. With the mechanical ankle, the axis is also estimated. Given that the mechanism is simpler and less variable than the natural joint, it is possible to place it closer to the true axis of the joint, ensuring the variations will be no greater than with a conventional model. Since the same device was used in all tests, the marker placement was the same for every run; thus, differences were only those generated by the changes to release timing.

\section{Statistical Analysis}

The data were filtered digitally using a sixth-order Butterworth filter, with a cutoff frequency of $6 \mathrm{~Hz}$. Statistical analysis was performed using the Tukey comparison method for general linear models.

\section{RESULTS}

The addition of the extra mass and linkage between the toes and the shank changed the way the device behaved. As the question being addressed in this study was effect of the delay, it is these changes that were studied. The majority of the results are for Subject 1 (unless indicated). Gait performance was studied for the unmodified prosthesis and for the device with five delay settings. The unmodified prosthesis was lighter than the modified one. After giving the subject time to adjust to the modified foot, the prosthesis was activated. At this point, the subject did confirm that with a delay the prosthesis could be felt giving a later push forward compared with no delay. In general, it was found that introducing a delay did affect prosthetic gait, but the effects were very small and not easily discernible in the data.

The introduction of the clutch slowed the release from $102 \%$ to $52 \%$. The peak in the ankle power generation (see "Kinetics" section p. 415) shows a lower but broader peak of power generation for the modified device compared with the unmodified one. 


\section{Verification}

With the foot aligned and the subject accustomed to the new prosthesis, the kinematics and kinetics of the system were recorded. Figure 6 illustrates the effect of delayed plantarflexion. The function of the design was verified by plotting the differential of the ankle angle with respect to time for no delay and for a delay of $85 \mathrm{~ms}$. The reduction in the ankle angular acceleration allowed the moment of transition between dorsiflexion and plantarflexion to be isolated. The area of interest is when the gradient tends to zero and shows no change in ankle angle. Figure 6 shows the delay in plantarflexion, which is characterized by a region with a much lower gradient.

\section{Kinematics}

The kinematic plots of the hip, knee, and ankle joints for both legs are illustrated in Figure 7. With these and subsequent plots, the results for the prosthetic side are on the left and the unaffected side are on the right. Following convention, the anatomical levels are plotted from top to bottom. The ranges of motion for each joint for both legs are listed in Table 1. The modified device introduced an increase of the range of motion of both hip joints. The absence of a delay at the ankle showed additional range at the left hip (prosthetic side) of up to $7^{\circ}$. This difference could be an artifact of the subject using the modified prosthesis and the increased inertia to

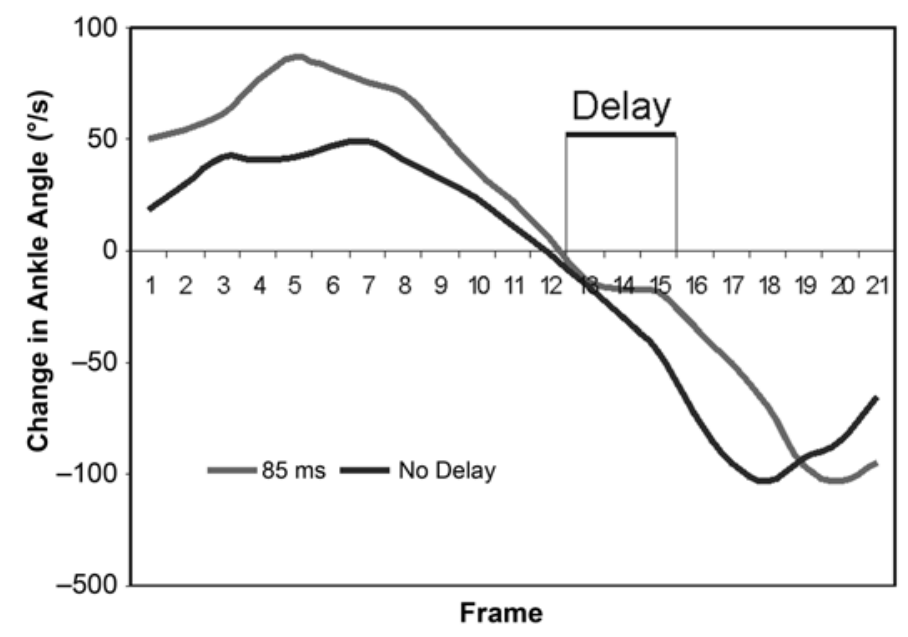

Figure 6.

Plot of differential of ankle flexion with respect to frame number (hence time) for Subject 1. Shown is No Delay and $85 \mathrm{~ms}$ delay, with region of low change in flexion that reflects delay of release of ankle. swinging the leg forward and backward. The differences at the right hip joint are less pronounced. The differences in the kinematics at the knee are most evident after TO, where the knee joint flexed to a maximum of $69.2^{\circ}$ with no delay and $75.3^{\circ}$ when the delay was introduced. This change in angle also delayed maximum knee flexion from 64 percent of the gait cycle to 72 percent, although the settings were identical in magnitude and timing. This delay could be a further artifact of the changes made to the foot. The largest change in joint kinematics was exhibited at the left ankle. Upon analysis, the most visible outcome of the trials was that without the added weight from the modifications, the prosthesis had a larger range of motion. The reduction in range of motion was attributed to decreased plantarflexion at foot contact. Although free spinning, the electromechanical brake did provide enough resistance to affect ankle kinematics by slowing its response to loads.

The ankle joint is a segment angle derived from the foot pitch and shank angle (Figure 8). The ankle's range of motion was found to vary greatly before and after modifications. Before the additional weight, the left ankle had a range of $20.25^{\circ} \pm 0.54^{\circ}$ but fell to between $8.44^{\circ} \pm 0.54^{\circ}$ and $14.85^{\circ} \pm 0.56^{\circ}$. Compared with no delay, all the delay settings promoted increased dorsiflexion, supporting the assertion that the delay had an impact on the performance of the device (significant difference at $p<0.05$ using Tukey comparison method for general linear models, with each trial an event).

\section{Kinetics}

The moment plots shown in Figure 9 illustrate how the joint moments were affected. The joint powers are displayed in Figure 10 and have been divided into zones corresponding to power generation and absorption events [2]. Compared with no delay, the left hip (prosthetic side) showed decreased extension moment during midstance. The left hip moment begins to approximate the right hip joint in both appearance and magnitude; this may result in increased comfort levels with the delayed plantarflexion. When the hip powers were derived, a similar result was observed with delayed plantarflexion approximating the subject's unaffected side. The largest difference in knee moments and powers between left and right was the lack of flexion at foot contact caused by the prosthesis's shockabsorbent foam. During stance phase, knee moment without modifications is virtually nonexistent. The low mass of the basic prosthesis produced smooth movement of the knee, while the additional weight caused an increased left 

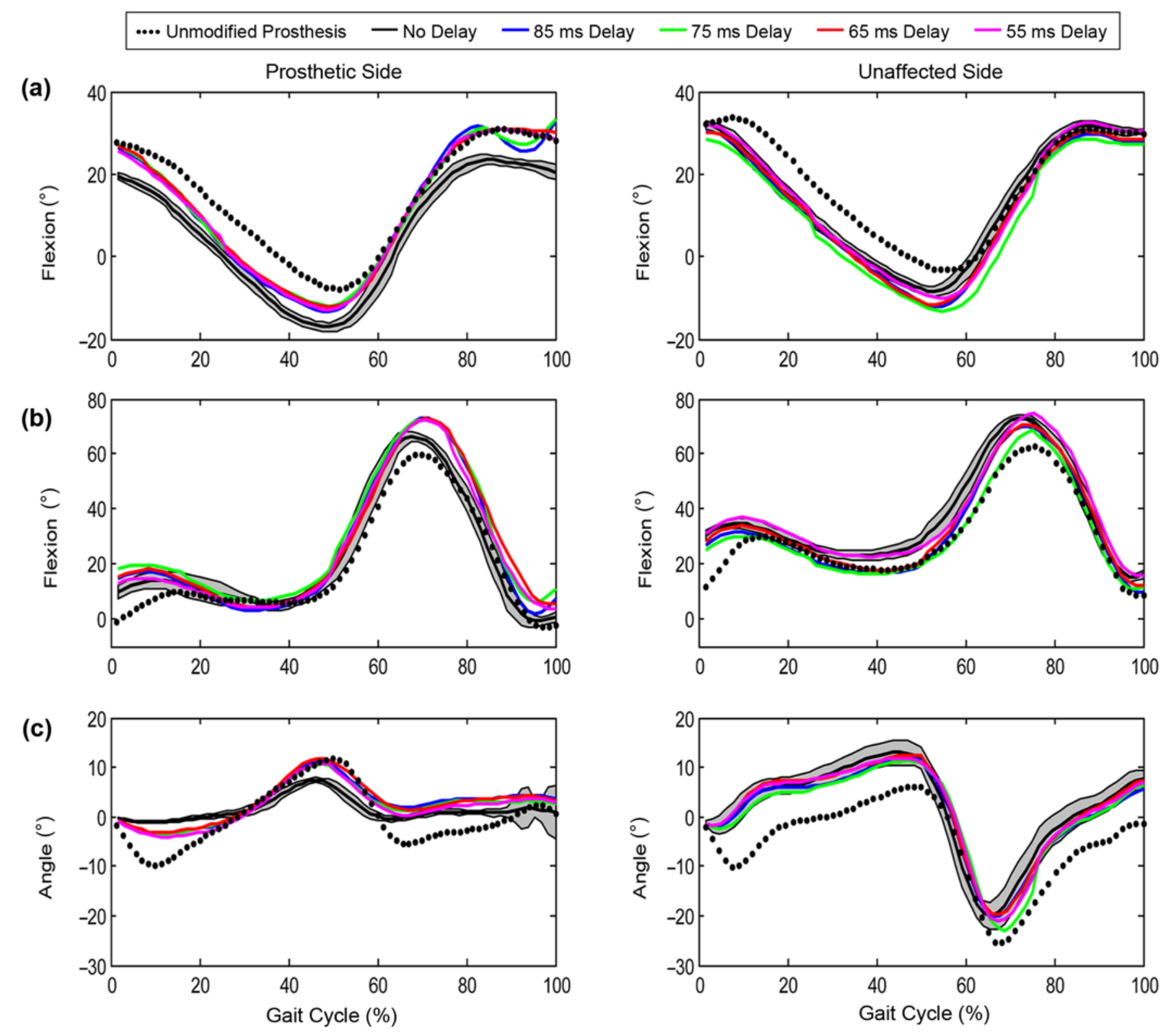

Figure 7.

Kinematic plots of (a) hip, (b) knee, and (c) ankle for Subject 1. Black dots indicate unmodified prosthesis. Black line is no delay and is shown with \pm 1 standard deviation error band around it. Color plots show impact of delay in releasing stored energy.

knee extension moment after TO. An additional moment was also needed to slow down the prosthesis after TO (after K3; from the convention in Winter [13]; peaks in energy storage or generation are indicated by numbers, $\mathrm{K}=$ knee). In relation to the right ankle moment plot, the prosthetic ankle was found to produce a maximum dorsiflexion moment less gradually and later in the gait cycle. There was a difference seen in the left ankle joint with no modifications, where the design forced the prosthesis to behave more similarly to the intact limb. No significant differences were found between passively generated power at no delay and delayed plantarflexion.

\section{Stride Characteristics}

The results derived from the stride characteristics are displayed in Table 2. A significant difference in gait velocity was perceived at 75 ms delay ( $p=0.04$, Tukey comparison method), where the subject slowed to 
Table 1.

Range of motion for each joint and setting, prosthetic (P) and sound (S), for Subject 1. Data presented as mean \pm standard deviation.

\begin{tabular}{|c|c|c|c|c|c|c|}
\hline \multirow{2}{*}{ Setting } & \multicolumn{2}{|c|}{ Hip ( ${ }^{\circ}$ ) } & \multicolumn{2}{|c|}{ Knee $\left(^{\circ}\right)$} & \multicolumn{2}{|c|}{ Ankle ( $\left.{ }^{\circ}\right)$} \\
\hline & L (P) & R (S) & $L(P)$ & R (S) & $L(P)$ & R (S) \\
\hline$\overline{85 \mathrm{~ms}}$ & $46.2 \pm 6.5$ & $42.6 \pm 1.6$ & $71.0 \pm 5.0$ & $60.3 \pm 2.4$ & $14.6 \pm 0.9$ & $31.8 \pm 3.5$ \\
\hline $75 \mathrm{~ms}$ & $45.2 \pm 6.4$ & $41.7 \pm 1.5$ & $66.3 \pm 4.6$ & $57.7 \pm 2.5$ & $14.0 \pm 0.8$ & $34.2 \pm 3.6$ \\
\hline $65 \mathrm{~ms}$ & $43.1 \pm 1.6$ & $41.9 \pm 1.8$ & $68.4 \pm 2.1$ & $58.6 \pm 2.3$ & $14.8 \pm 0.6$ & $32.4 \pm 3.0$ \\
\hline $55 \mathrm{~ms}$ & $43.7 \pm 1.4$ & $42.5 \pm 1.5$ & $68.5 \pm 2.6$ & $58.9 \pm 2.6$ & $14.8 \pm 0.8$ & $33.0 \pm 3.9$ \\
\hline No Delay & $40.6 \pm 1.7$ & $40.2 \pm 1.5$ & $67.1 \pm 2.6$ & $58.1 \pm 1.6$ & $8.4 \pm 0.5$ & $33.0 \pm 3.7$ \\
\hline
\end{tabular}

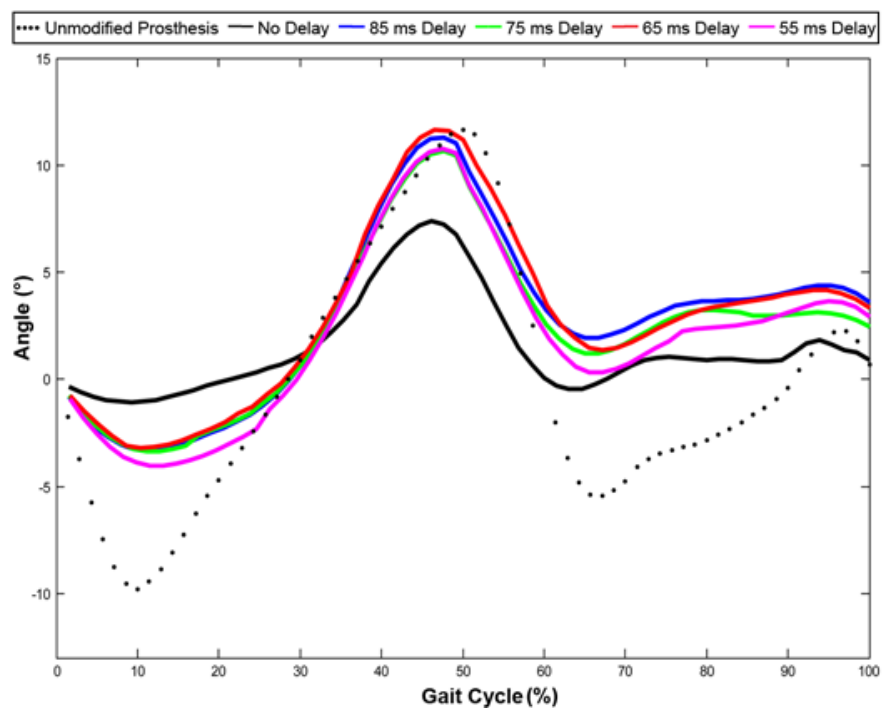

Figure 8.

Plot of ankle angle for Subject 1. Black dots indicate unmodified prosthesis. Black line is no delay and is shown with \pm 1 standard deviation error band around it. Color plots show impact of delay in releasing stored energy.

$1.22 \mathrm{~m} / \mathrm{s}$. The mean gait velocity for all settings was found to be $1.28 \mathrm{~m} / \mathrm{s}$. Cadence describes gait cycle in steps per minute. The cadence deviation for all settings was found to be $<2.0$ steps/min. With the exception of 75 ms and unmodified (where it was significant at the $p<$ 0.01 level), no significant differences were found between delay settings. Ideally, in nondisabled balanced gait, the difference between step length in the left and right legs is small. A large difference in step length is a clear indicator of imbalanced gait. After the system was installed and delayed plantarflexion was introduced, no significant differences in step length were distinguished (minimum $p$-value $=0.09$ at $55 \mathrm{~ms}$, Tukey comparison method).
Related to step length, the stride length provides a global view of gait symmetry. There was no significant difference in stride length found between before and after the modifications. The difference in stride length was seen to be $2.0 \mathrm{~cm}$ compared with $7.0 \mathrm{~cm}$ with the extra mass (minimum $p$-value $=0.10$ at $65 \mathrm{~ms}$ ). In addition, there was no significant difference found between delay settings.

\section{Impact of Design}

The design changed the manner in which the foot released the energy. This was different from the way it released energy in the unmodified form. It was similar for any of the release timings in the modified device. A numerical study of one stride in each of the 10 walks for each of the release timings for Subject 1 was performed. At release, the change in angle between the shank and the toes was recorded, and it showed a release rate of $52.0 \% \pm 7 \%$; an analysis of variance of all the data did not reject the hypothesis that they were all the same population ( $p$-value $=$ 0.12 with $d f=49$, for 5 categories: $0,55,65,75$, and $85 \mathrm{~ms}$ delay).

\section{Subjective Assessment}

In daily testing, Subject 1 reported preferring a $65 \mathrm{~ms}$ delay as the setting at which gait was the most comfortable. However, during the final session, when the different settings were blinded and presented in a random order, the subject reported that the 85 ms delay was the most comfortable. Delays shorter than $65 \mathrm{~ms}$ were reported to be more uncomfortable than longer ones.

Subject 2 adapted well to the foot and could detect the change in the delays. He determined that his favored delay was $55 \mathrm{~ms}$. He also consistently felt that it would be appropriate to walk slower with the longer delays, saying "Almost makes me feel like I want to go slower, kind of like an 'easy stroll' setting.” 
(a)

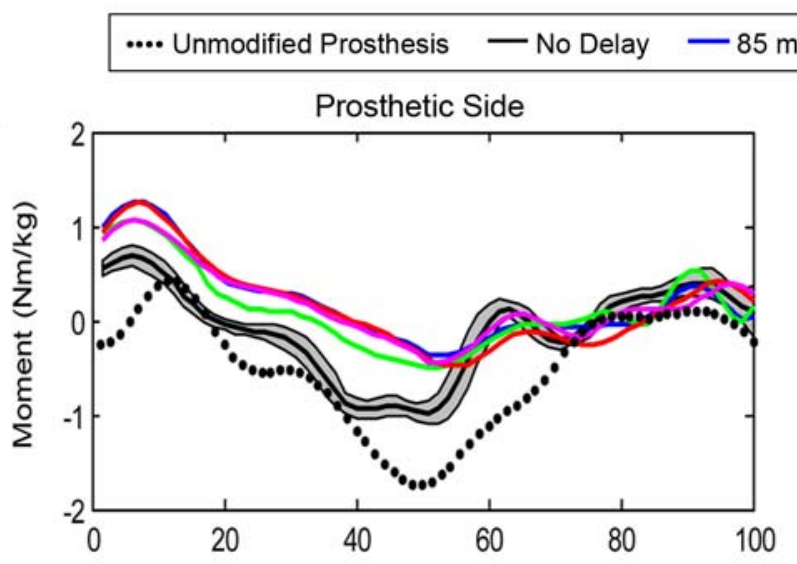

(b)
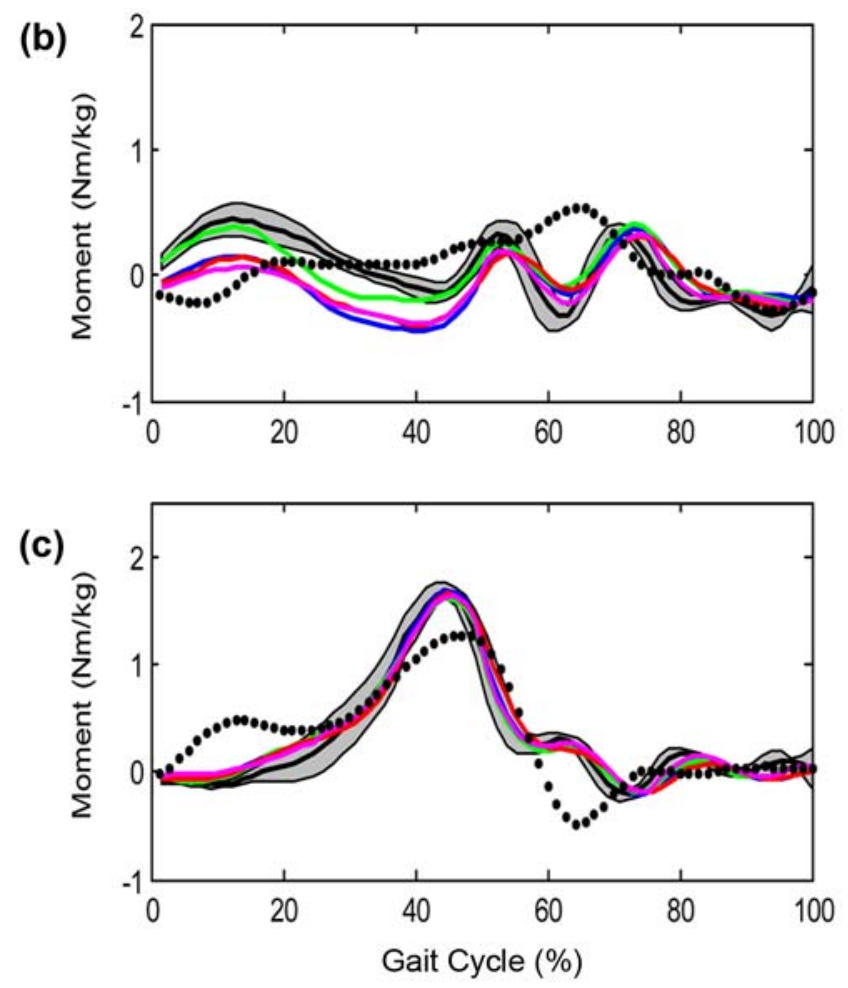

$-75 \mathrm{~ms}$ Delay $-65 \mathrm{~ms}$ Delay $-55 \mathrm{~ms}$ Delay
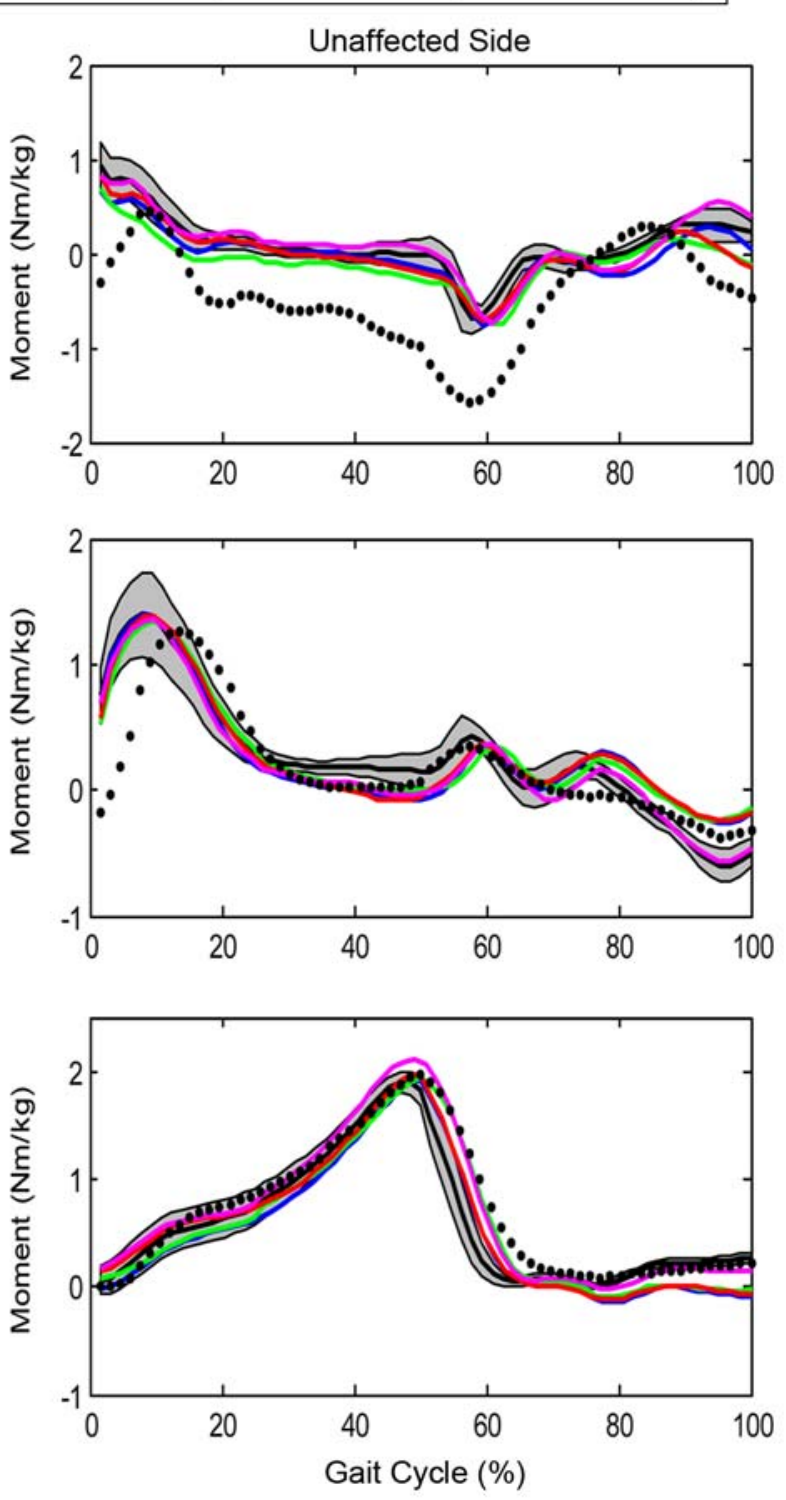

Figure 9.

Kinetic moment plots of (a) hip, (b) knee, and (c) ankle for Subject 1. Black dots indicate unmodified prosthesis. Black line is no delay and is shown with \pm 1 standard deviation error band around it. Color plots show impact of delay in releasing stored energy.

\section{DISCUSSION}

The moments at the ankle were altered by the delayed release (Figure 9); they increased from $1.3 \mathrm{Nm} /$ $\mathrm{kg}$ to $1.75 \mathrm{Nm} / \mathrm{kg}$, which is substantially closer to the performance of the sound limb $(1.9 \mathrm{Nm} / \mathrm{kg})$. This change to a form closer to the unaffected side is reflected throughout the leg. The ankle plantarflexes more slowly as well as releasing the energy more slowly (Figure 7). On the prosthetic side, the knee is then able to deliver more power to the leg later on in the stride $(1 \mathrm{~W} / \mathrm{kg}$ compared with $0.4 \mathrm{~W} / \mathrm{kg}$ ), so the hip has to deliver less power (1 W/kg as opposed to $2 \mathrm{~W} / \mathrm{kg}$ ) (Figure 10). This may make the prosthesis feel more comfortable.

In general, when the increased mass of the brake mechanism is added to the foot, both the performance of 
(a)

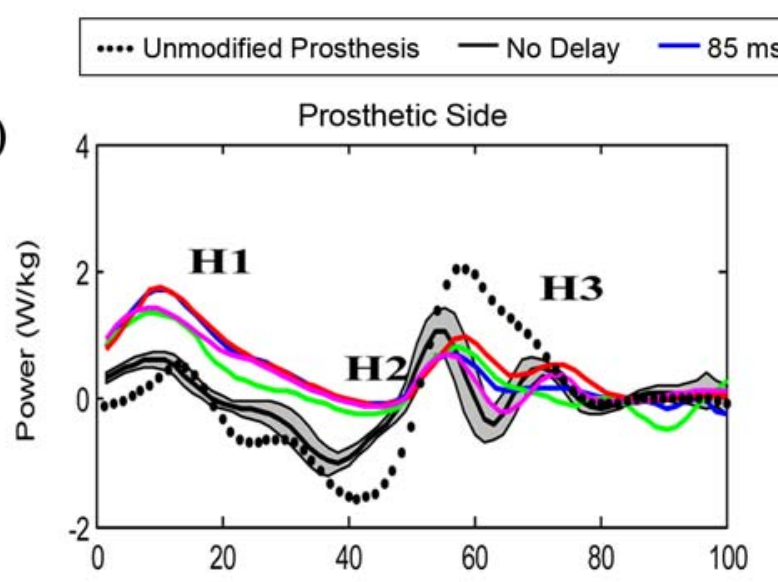

(b)

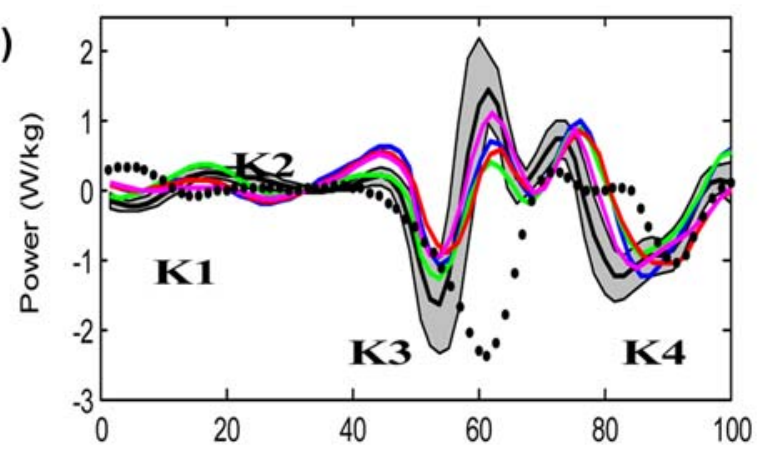

(c)

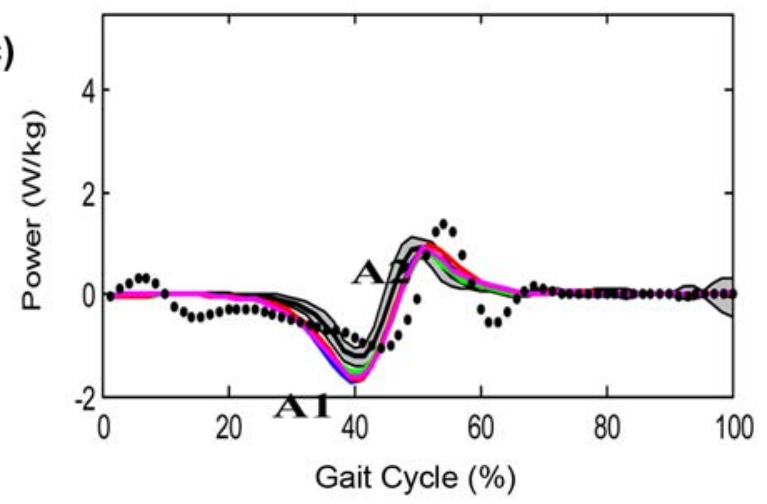


Table 2.

Stride characteristics for Subject 1. Data presented as mean \pm standard deviation.

\begin{tabular}{|c|c|c|c|c|c|c|}
\hline Parameter & $85 \mathrm{~ms}$ & $75 \mathrm{~ms}$ & $65 \mathrm{~ms}$ & $55 \mathrm{~ms}$ & No Delay & Unmodified \\
\hline Gait Velocity (m/s) & $1.28 \pm 0.03$ & $1.22 \pm 0.03$ & $1.28 \pm 0.03$ & $1.20 \pm 0.18$ & $1.28 \pm 0.04$ & $1.25 \pm 0.02$ \\
\hline Cadence (steps/min) & $107.0 \pm 1.5$ & $102.0 \pm 2.0^{*}$ & $109.0 \pm 2.1$ & $107.0 \pm 3.4$ & $106.5 \pm 1.3$ & $98.0 \pm 0.4^{*}$ \\
\hline Step Length R:L (m) & $0.75 \pm 0.02$ & $0.77 \pm 0.03$ & $0.75 \pm 0.02$ & $0.79 \pm 0.02$ & $0.76 \pm 0.03$ & $0.80 \pm 0.01$ \\
\hline Stride Length: R (m) & $1.53 \pm 0.03$ & $1.49 \pm 0.03$ & $1.53 \pm 0.04$ & $1.53 \pm 0.03$ & $1.51 \pm 0.04$ & $1.49 \pm 0.01$ \\
\hline Stride Length: L (m) & $1.45 \pm 0.03$ & $1.45 \pm 0.03$ & $1.44 \pm 0.03$ & $1.47 \pm 0.04$ & $1.46 \pm 0.02$ & $1.51 \pm 0.02$ \\
\hline
\end{tabular}

*Significant values.

$\mathrm{L}=$ left, $\mathrm{R}=$ right.

to see whether the other designs that can generate power will be able to restore more natural motion and behavior.

Figures 9 and $\mathbf{1 0}$ show the release of the power being advanced relative to the unmodified device. This is likely to be an artifact of the process of gait analysis, which fits different length strides together on the same scale. As the unaffected stride has a longer step length, the release point is pushed backward relative to the shorter stride of the affected limb.

\section{CONCLUSIONS}

This study examined the effects of delayed plantarflexion on persons with lower-limb loss. It was found that the combination of doubling the mass of the prosthesis and tethering the shank and ankle did have an effect on the gait cycle of both limbs, with the ranges of motion and timing of the stride becoming closer to the nondisabled population. After all delay settings were tested, subject 1 found that the 65 ms delay was the most comfortable. The wearer did feel a distinctive delayed push after each step, but this could not be confirmed statistically. Subject 2 did feel that the longer delay was appropriate for a slower walking speed, which suggests that an ankle that responds to the user's pace may be useful in a similar manner as the adaptive swing-phase knee designs that are available. Based on the statement of the subjects and the data, it would appear that lengthening the impulse from the foot makes it more comfortable to use. The next stages of work would include a reduction of the design mass to enable the device to be used in the field.

\section{ACKNOWLEDGMENTS}

Author Contributions:

Design, construction, experiment, analysis, writing: M. Mitchell.
Experiment, analysis: K. Craig.

Supervision of design, construction, experiment, analysis, and writing: P. Kyberd, E. Biden.

Assist in design of device and experiment, fitting prosthesis, clinical supervision, and comment on writing: G. Bush.

Financial Disclosures: The authors have declared that no competing interests exist.

Funding/Support: This material was based on work supported by the Canadian Foundation for Innovation, Canadian Institutes of Health Research (grant IEG-36878); Canada Research Chairs Program, Natural Sciences and Engineering Research Council of Canada (grant 312094-05); and the New Brunswick Innovation Foundation start-up program in "Prosthetics Research."

Institutional Review: Testing was approved by the University of New Brunswick Institutional Review Board (approval number 2005-059).

Participant Follow-Up: The participants are already aware of the results and the publication process; this forms part of the University of New Brunswick Institutional Review Board process. They will be provided with a copy of the article (via their prosthetists) when it is available.

Additional Contributions: The authors would like to thank various organizations that have supported this work: Canadian Foundation for Innovation, Canadian Institutes of Health Research; Canada Research Chairs Program, Natural Sciences and Engineering Research Council of Canada; New Brunswick Innovation Foundation; and the participants for their time and patience.

\section{REFERENCES}

1. Hafner BJ, Sanders JE, Czerniecki JM, Fergason J. Energy storage and return prostheses: does patient perception correlate with biomechanical analysis? Clin Biomech (Bristol, Avon). 2002;17(5):325-44. [PMID:12084537] http://dx.doi.org/10.1016/S0268-0033(02)00020-7

2. Winter DA, Sienko SE. Biomechanics of below-knee amputee gait. J Biomech. 1988;21(5):361-67.

[PMID:3417688] http://dx.doi.org/10.1016/0021-9290(88)90142-X

3. Boda W, Findley T, Tapp W, Reisman S, Lacker M, Chaudry H, Sisto S, Cordero D, Davis A. Comparison of normal and amputee gait with added weight. In: Reisman 
SS, editor. Proceedings of the Nineteenth IEEE Annual Bioengineering Conference; 1993 Mar 18-19; Newark, New Jersey. Piscataway (NJ): IEEE; 1993. p. 217-18.

4. Perry J. Normal gait. In: Smith DG, Michael JW, Bowker $\mathrm{JH}$, editors. Atlas of amputations and limb deficiencies. 3rd ed. Rosemont (IL): American Academy of Orthopaedic Surgeons; 2004. p. 353-66.

5. Perry J. Amputee gait. In: Smith DG, Michael JW, Bowker $\mathrm{JH}$, editors. Atlas of amputations and limb deficiencies. 3rd ed. Rosemont (IL): American Academy of Orthopaedic Surgeons; 2004. p. 367-84.

6. Sutherland DH, Olshen RA, Biden EN, Wyatt MP. The development of mature walking. Oxford (UK): Blackwell Scientific Publications Ltd; 1988.

7. Burgess EM, Poggi DL, Hittenberger DA, Zettl JH, Moeller DE, Carpenter KL, Forsgren SM. Development and preliminary evaluation of the VA Seattle foot. J Rehabil Res Dev. 1985;22(3):75-84. [PMID:3835268] http://dx.doi.org/10.1682/JRRD.1985.07.0075

8. Czerniecki JM, Gitter A, Munro C. Joint moment and muscle power output characteristics of below knee amputees during running: the influence of energy storing prosthetic feet. J Biomech. 1991;24(1):63-75. [PMID:2026634] http://dx.doi.org/10.1016/0021-9290(91)90327-J

9. Ehara Y, Beppu M, Nomura S, Kunimi Y, Takahashi S. Energy storing property of so-called energy-storing prosthetic feet. Arch Phys Med Rehabil. 1993;74(1):68-72. [PMID:8420524]

10. Geil MD. Effectiveness evaluation and functional theoretical modeling of dynamic elastic response lower limb prosthetics [dissertation]. [Columbus (OH)]: Ohio State University; 1997.

11. Sadeghi H. Contributions of lower-limb muscle power in gait of people without impairments. Phys Ther. 2000; 80(12):1188-96. [PMID:11087305]

12. Sup F, Bohara A, Goldfarb M. Design and control of a powered transfemoral prosthesis. Int J Rob Res. 2008; 27(2):263-73. [PMID:19898683]

http://dx.doi.org/10.1177/0278364907084588

13. Winter DA. The biomechanics of motor control of human gait: Normal, elderly and pathological. 2nd ed. Waterloo (Canada): University of Waterloo Press; 1991.

14. Klute GK, Czerniecki J, Hannaford B. Development of powered prosthetic lower limb. In: Proceedings 1st National Meeting, Department of Veterans Affairs Rehabilitation Research and Development Service; 1998 Oct; Washington (DC): D6. Available from: http://www.rehab.research.va.gov/va/98/oktdemos.htm.

15. Kyberd P. The intelligent hand. IEE Rev. 2000;46(5):31-35. http://dx.doi.org/10.1049/ir:20000508

16. Au S, Weber J, Herr H. Powered ankle-foot prosthesis improves walking metabolic economy. IEEE Trans Robot. 2009;25(1):51-66. http://dx.doi.org/10.1109/TRO.2008.2008747
17. Bellman RD, Holgate MA, Sugar TG. SPARKy 3: Design of an active robotic ankle prosthesis with two actuated degrees of freedom using regenerative kinetics. In: Proceedings of the 2nd Biennial IEEE/RAS-EMBS International Conference on Biomedical Robotics and Biomechatronics; 2008 Oct 19-22; Scottsdale, Arizona. Piscataway (NJ): IEEE; 2008.

18. Collins SH, Kuo AD. Recycling energy to restore impaired ankle function during human walking. PLoS ONE. 2010; 5(2):e9307. [PMID:20174659] http://dx.doi.org/10.1371/journal.pone.0009307

19. Segal AD, Zelik KE, Klute GK, Morgenroth DC, Hahn ME, Orendurff MS, Adamczyk PG, Collins SH, Kuo AD, Czerniecki JM. The effects of a controlled energy storage and return prototype prosthetic foot on transtibial amputee ambulation. Hum Mov Sci. 2012;31(4):918-31. [PMID:22100728] http://dx.doi.org/10.1016/j.humov.2011.08.005

20. Zelik KE, Collins SH, Adamczyk PG, Segal AD, Klute GK, Morgenroth DC, Hahn ME, Orendurff MS, Czerniecki JM, Kuo AD. Systematic variation of prosthetic foot spring affects center-of-mass mechanics and metabolic cost during walking. IEEE Trans Neural Syst Rehabil Eng. 2011; 19(4):411-19. [PMID:21708509] http://dx.doi.org/10.1109/TNSRE.2011.2159018

21. Selles RW, Bussmann JB, Klip LM, Speet B, Van Soest AJ, Stam HJ. Adaptations to mass perturbations in transtibial amputees: kinetic or kinematic invariance? Arch Phys Med Rehabil. 2004;85(12):2046-52. [PMID:15605346] http://dx.doi.org/10.1016/j.apmr.2003.10.013

22. Royer TD, Martin PE. Manipulations of leg mass and moment of inertia: effects on energy cost of walking. Med Sci Sports Exerc. 2005;37(4):649-56. [PMID:15809565] http://dx.doi.org/10.1249/01.MSS.0000159007.56083.96

23. Mattes SJ, Martin PE, Royer TD. Walking symmetry and energy cost in persons with unilateral transtibial amputations: matching prosthetic and intact limb inertial properties. Arch Phys Med Rehabil. 2000;81(5):561-68. [PMID:10807092] http://dx.doi.org/10.1016/S0003-9993(00)90035-2

24. Rusaw D, Ramstrand N. Motion-analysis studies of transtibial prosthesis users: a systematic review. Prosthet Orthot Int. 2011;35(1):8-19. [PMID:21515885] http://dx.doi.org/10.1177/0309364610393060

25. Gailey RS, Wenger MA, Raya M, Kirk N, Erbs K, Spyropoulos P, Nash MS. Energy expenditure of trans-tibial amputees during ambulation at self-selected pace. Prosthet Orthot Int. 1994;18(2):84-91. [PMID:7991365]

Submitted for publication June 10, 2011. Accepted in revised form July 24, 2012. 
JRRD, Volume 50, Number 3, 2013

This article and any supplemental material should be cited as follows:

Mitchell M, Craig K, Kyberd P, Biden E, Bush G. Design and development of ankle-foot prosthesis with delayed release of plantarflexion. J Rehabil Res Dev. 2013;50(3):
409-22.

http://dx.doi.org/10.1682/JRRD.2011.06.0107

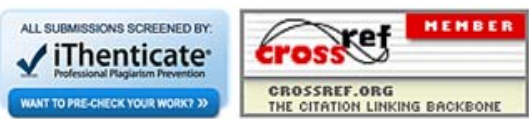

